\title{
TREATMENT OF PAINFUL CALICEAL STONES
}

\author{
T. A. COURY, M.D. \\ L. PAUL SONDA, M.D. \\ J. E. LINGEMAN, M.D. \\ R. J. KAHNOSKI, M.D.
}

From Methodist Hospital of Indiana Institute for Kidney Stone Disease, Department of Medical Research, Indianapolis; University of Michigan Medical Center, Department of Surgery, Section of Urology, Ann Arbor; and Indiana University School of Medicine, Indianapolis, Indiana

\begin{abstract}
Nonmobile caliceal stones cause pain more often than previously appreciated. The character and intensity of the pain differs from typical renal colic. Twenty-six patients with caliceal stones and pain underwent attempted treatment for pain control via stone removal or disintegration: 15 were treated with percutaneous stone extraction (PSE), 10 with extracorporeal shock-wave lithotripsy (ESWL), and I required open surgery after failing PSE. One patient had persistent pain after ESWL and subsequently underwent PSE; 25 of 26 patients had complete relief of pain. Morbidity was minimal. Patients with painful caliceal stones should be offered ESWL, followed by PSE if pain persists.
\end{abstract}

Simple caliceal stones, documented to be immobile, have been believed to be an unlikely explanation for a patient's subjective complaint of flank or anterior abdominal pain. Many of these patients were thought to have nonrenal pain leading to extensive radiologic studies and consultation with numerous physicians seeking an etiology for the problem. Even when no etiology was found, reluctance to perform surgery persisted due to the potential difficulty and morbidity of the procedure and doubt as to whether or not pain would be relieved. The introduction of percutaneous stone extraction (PSE) and extracorporeal shock-wave lithotripsy (ESWL) has provided a less morbid, technically simple means of attempting to alleviate their pain.

The urology departments at the University of Michigan Hospitals and Methodist Hospital of Indiana began stone removal via PSE or disintegration with ESWL for these patients. Twentysix patients with pain and nonmobile caliceal stones were treated with these new techniques in the one-year period January to December, 1984.
Material, Methods, and Results

Twenty-six patients were identified retrospectively, 12 from University of Michigan Hospital and 14 from Methodist Hospital of Indiana. The patients had been evaluated by two primary investigators (LPS and JEL) with respect to stone size, location, and pain. After the investigators were convinced that the patient's pain was likely secondary to the stone and debilitating enough to merit intervention, either PSE or ESWL were used to treat the patients. PSE was performed primarily as a twostage procedure under local anesthesia (UMH) or in a single stage under general anesthesia (MHI). ESWL was performed as a single stage procedure using the Dornier $\mathrm{HM}^{3}$ Lithotriptor. ${ }^{1}$ All patients were contacted or had been seen for follow-up at least one year after their treatment.

Twenty-six patients (average age $47 \mathrm{yrs}$ ) with 32 nonmobile caliceal stones had 28 procedures to remove the stones. There were 12 males and 14 females. The average stone size was $9.1 \mathrm{~mm}$ varying from $2-20 \mathrm{~mm}$. Forty-six per cent of stones were in the left kidney and 54 per cent in 


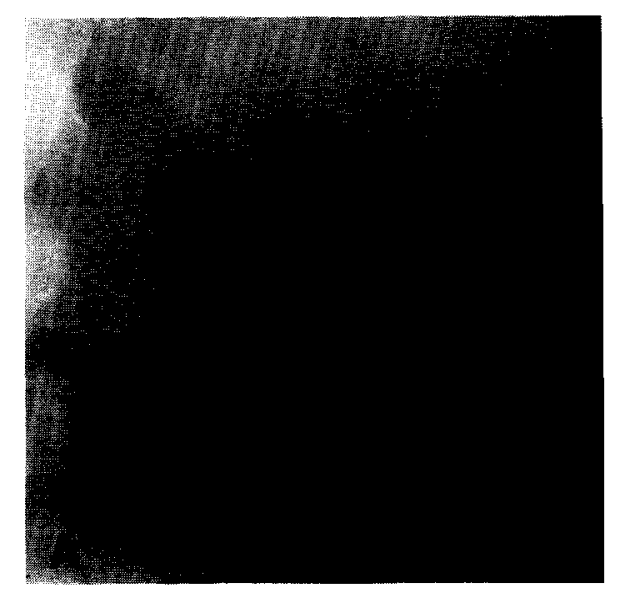

FIGURE 1. Case 1. (A) KUB pretreatment shows left lower caliceal stone (solitary left kidney); (B) IVP pretreatment demonstrating no obstruction; and $(C) K U B$ three-months after ESWL shows patient to be stone-free.
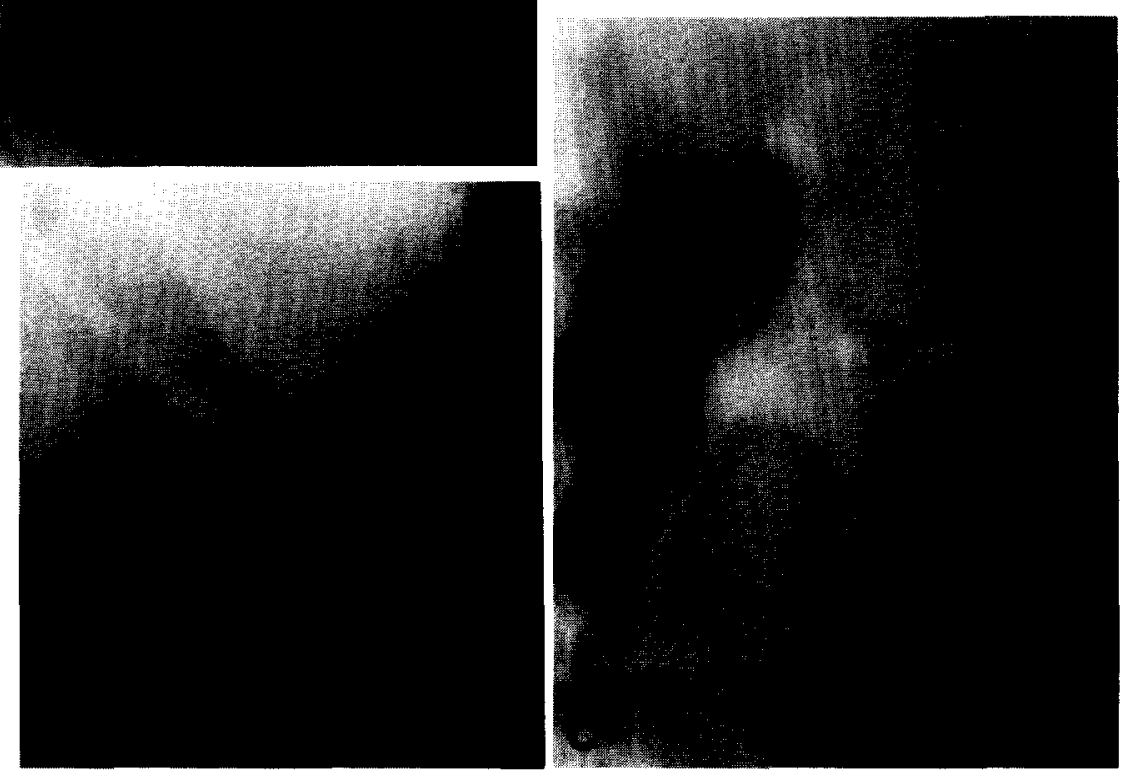

the right. Twenty-three patients had solitary stones: 18 lower caliceal $(69 \%), 3$ middle $(12 \%)$, and 2 upper $(7 \%)$. Three patients had multiple caliceal stones $(12 \%)$. Twenty-seven per cent $(7 / 26)$ of the patients had extensive medical evaluation including gastrointestinal $x$ ray films, computerized tomography (CT) scans, and nonurologic consultation prior to stone surgery. The average length of time with pain was 2.5 years (range 3 months to 14 years). Fifty-eight per cent (15/26) had previous stone passage or stone surgery. The amount of pain preoperatively ranged from mild in $9(35 \%)$ to moderate (oral narcotics) in $13(50 \%)$ to severe (parenteral narcotics) in $3(11 \%)$.

There were 16 PSE, 11 ESWL, and 1 open operation. One ESWL failed to fragment the stone, requiring PSE, and one PSE failed due to access problems in a patient whose stone was in an anterior calix with a narrowed infundibulum, requiring open surgery. Twenty-five of 26 patients became pain-free with stone removal. The other patient had temporary relief of pain (3 months).

\section{Case 1}

\section{Case Reports}

A forty-three-year-old white woman presented with a solitary left kidney (previous nephrectomy for stones) and a $6 \times 8 \mathrm{~mm}$ stone in a lower calix (Fig. 1A and B). She had had pain for two years requiring intermittent oral codeine. In March, 1984, she underwent ESWL and received 1,100 shock waves at 20 $\mathrm{kV}$. She was discharged on the third postoperative day. Follow-up at twelve months revealed patient to be stone- and pain-free (Fig. 1C).

\section{Case 2}

A fifty-five-year-old white woman had a long history of renal calculi. She had had three open surgical procedures for stones in the past. Since 1970, she experienced significant pain in the costovertebral angle (CVA) and right upper quadrant associated with a $7 \times 10$ immobile right lower calix stone (Fig. 2A). During this time, she had a full medical workup for the pain which was negative. An intravenous pyelogram (IVP) showed no obstruction (Fig. 2B). She was seen by several urologists but was not treated because of the size, location, and noncolicky nature of the pain. In April, 1984, she was treated with ESWL, receiving 1,200 shockwaves at $24 \mathrm{kV}$. The stone did not fragment, and pain continued (Fig. 2C). Subsequently in July, 1984, she had PSE removing the stone successfully (Fig. 2D). Follow-up in March, 1985, revealed the patient to be stone- and pain-free. 
Figure 2. Case 2. (A) KUB pretreatment demonstrating right lower caliceal stone; (B) IVP pretreatment shows no obstruction; and (C) KUB following ESWL shows little change in calculus; patient still symptomatic; (D) KUB forty-eight hours after nephrostolithotomy demonstrating complete stone removal.
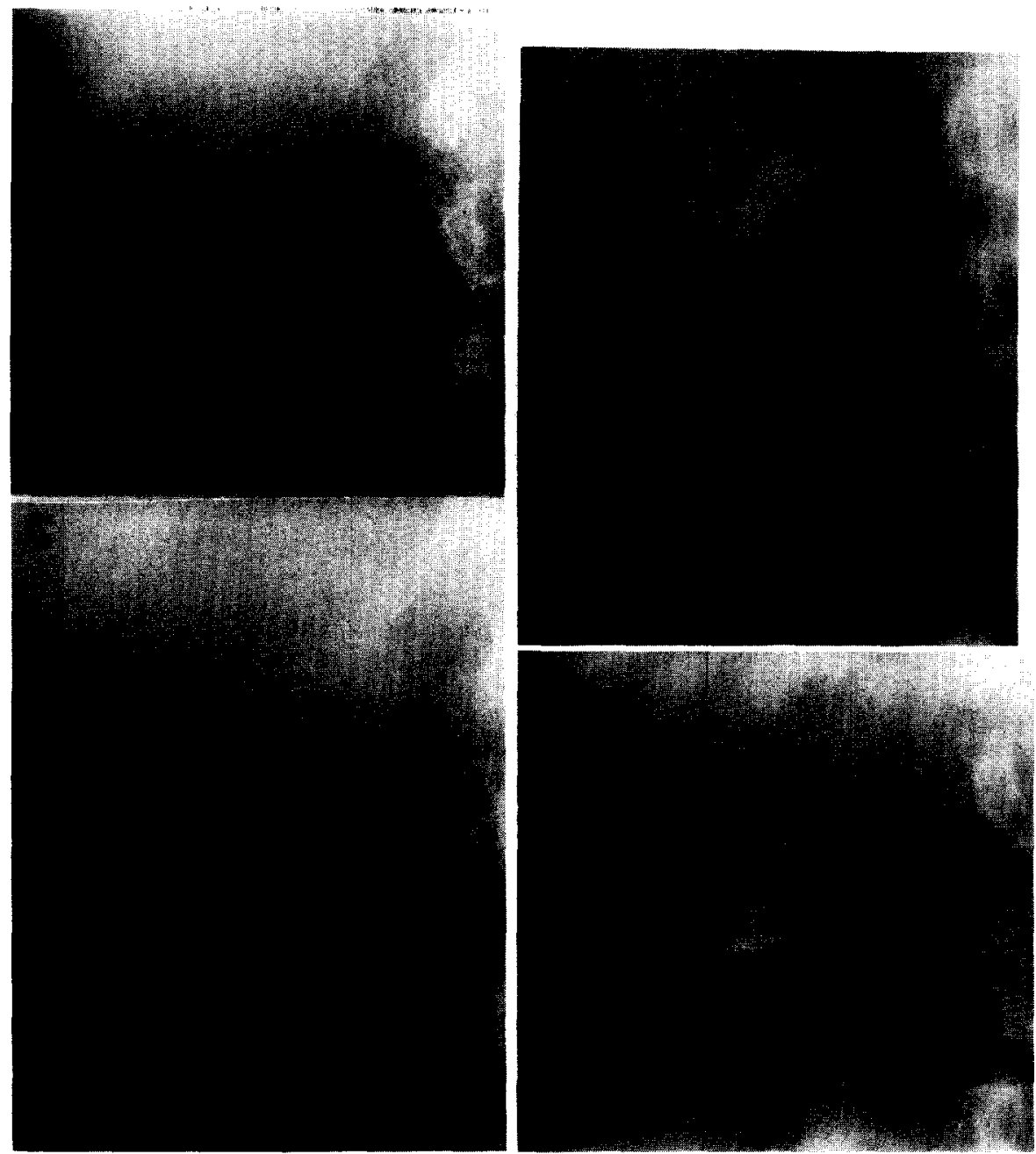

\section{Case 3}

A forty-six-year-old white woman had left flank pain and was unable to work (late January, 1984) until her admission to another hospital (March 5, 1984) where oral narcotics failed to relieve her pain. IVP, retrograde pyelograms, and serial kidney-ureter-bladder (KUB) films showed a 3-mm calculus in the left lower pole calix (Fig. 3A and B). The stone did not move on several films. She was transferred (March $21,1984)$, and medical evaluation showed no other source for pain. The patient received persistent parenteral narcotics and was eventually discharged on oral medication for another trial of conservative management. The stone was eventually removed percutaneously (April 23, 1984). Within days her pain was gone, and she is currently not taking pain medication and working full-time.

\section{Case 4}

A forty-three-year-old white woman had recurrent nephrolithiasis for six years. In 1978,

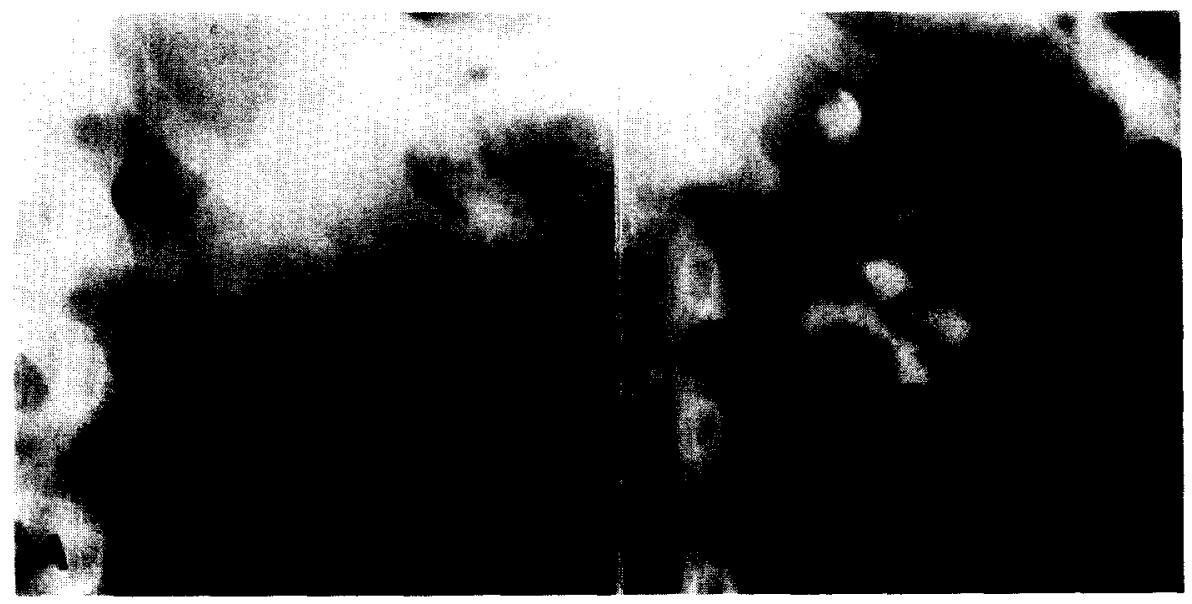

Figure 3. Case 3. (A) KUB pretreatment reveals a $3 \mathrm{~mm}$ left lower pole renal calculus; (B) retrograde pyelogram shows no obstruction. 
Figure 4. Case 4. (A) KUB pretreatment shows two right renal caliceal stones; (B) retrograde pyelogram demonstrating no obstruction.

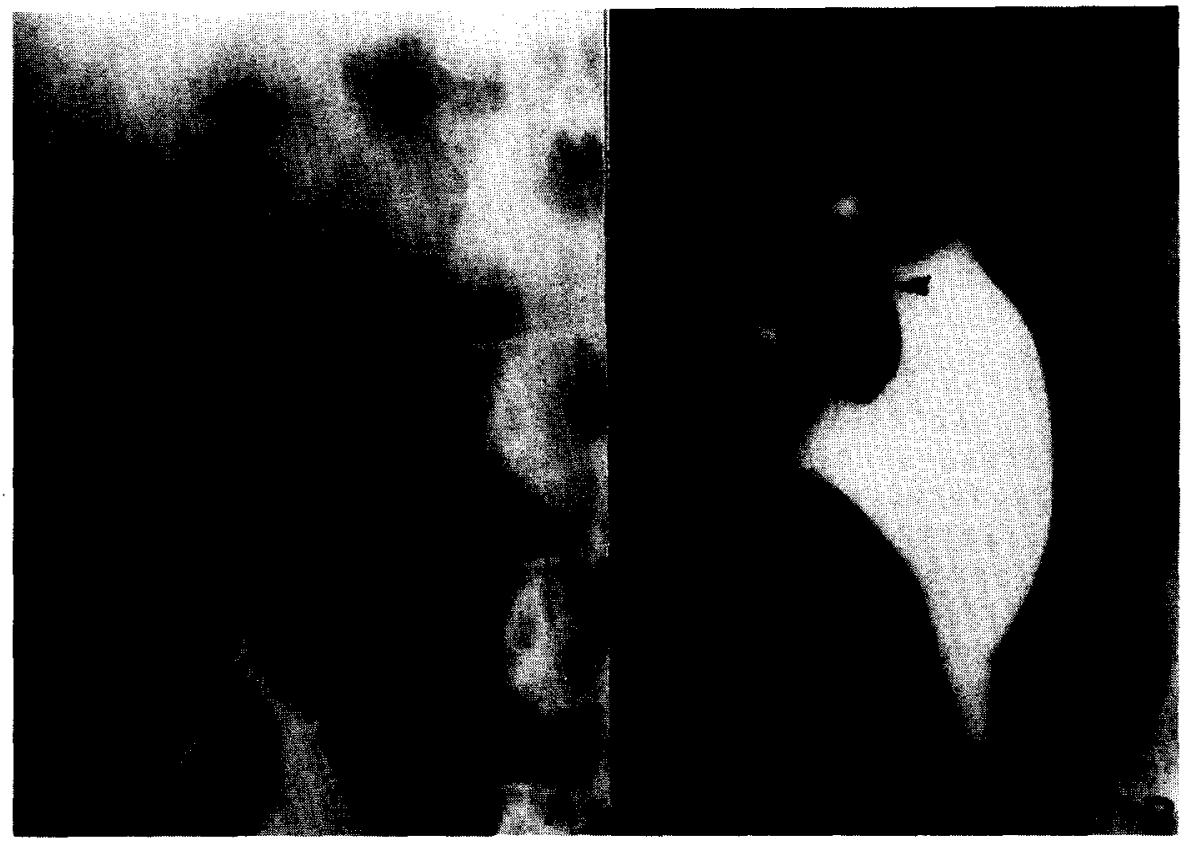

she underwent bilateral nephrolithotomy. In 1982, she underwent bilateral ureteral lithotomy and a repeat left nephrolithotomy. Pain on her left side was gone despite the presence of three residual calculi. The pain on her right side required eight to twelve analgesic tablets (Percodan) daily for over one year. She had interstitial nephritis with bilateral papillary necrosis and a serum creatinine of $2.7 \mathrm{mg} / 100 \mathrm{~mL}$. The right kidney had two stones, each $1 \mathrm{~cm}$, in caliceal positions, and never moving on multiple plain films (Fig. 4A and B). On May 13, 1984, percutaneous stone extraction was performed. Postoperatively, a delayed perinephric hematoma developed, which was treated conservatively and resolved by ultrasound completely one month later. By that time, she was not taking any pain pills and has done well since.

\section{Comment}

Caliceal stone pain is generally not typical renal colic. The pain usually does not radiate, but rather presents directly over the kidney and is constant, rather than spasmodic or episodic. It may be vague and atypical or even presenting rarely as anterior pain. For these reasons, it can be confused with pain of musculoskeletal or gastrointestinal (GI) origin. The pain can be worsened by activity with resultant confusion about a possible musculoskeletal source. Twenty-seven per cent of our patients had other medical evaluation, primarily GI studies. Pain was usually not severe. Fifty per cent of patients required oral narcotics, and rarely, admission to the hospital and parenteral pain medication were required. Paitents were persistent enough that they continued to seek medi- cal attention. The chronicity of the pain is underscored by the fact that the average duration of pain was two and a half years. Fifty-eight per cent had previous stone passage or surgery, suggesting that these patients were predisposed to having a psychogenic stimulus for their pain. Having already experienced either renal colic or postoperative pain, these patients were well aware of the pain they might experience should their immobile caliceal stones begin to pass, thus magnifying whatever discomfort they were experiencing. While this phenomenon may have existed and led to circumstances such as the patients being admitted for parenteral medication, it cannot entirely explain the uniformly good results which resulted, particularly in the other patients who never previously had had stones. We conclude that in some circumstances, immobile caliceal stones can be the primary source of pain.

The pathophysiology of renal pain has been described. ${ }^{2-7}$ Renal pain fibers follow sympathetic pathways via the celiac plexus, thoracic and upper lumbar splanchnic nerves, and abdominal aortic plexus. They reach the spinal cord from $T_{1}$ and $L_{2}$ dorsal spinal nerve roots. ${ }^{3}$ Dewolf and Fraley ${ }^{2}$ subdivided renal pain into visceral, colic, and referred. Colic is related to acute distention of upper urinary tract above the distal ureter. Referred pain relates to the patient's perception of pain at a more distant area of the body due to the common nerve supply. Visceral pain, a more persistent but less severe, aching discomfort, is the most common type associated with caliceal stones. Visceral pain is perceived in the lateral lumbar area at or below the eleventh and twelfth ribs. Electrical stimulation, pressure, or stretch of peripelvic renal 
capsule, pelvis, or vessels elicit visceral pain. ${ }^{8}$ The sympathetic nerves to the kidney can theoretically be blocked by injection or local anesthesia or by thoracolumbar sympathectomy. No nerve blocks were attempted on these patients to confirm renal origin of their pain.

Most patients with caliceal stones which are documented as immobile do not have pain. Previously, the authors were skeptical of patients complaining of pain with stable and immobile caliceal stones. Open surgical procedures to identify and remove stones in an intracaliceal position was also potentially difficult often requiring nephrotomy. With the advent of both percutaneous nephrostolithotomy and extracorporeal shock-wave lithotripsy, treating these stones is simplified and appears almost uniformly rewarding. Andersson and Sylven ${ }^{8}$ and Brannen, Busch, and Lewis ${ }^{10}$ have reported percutaneous removal of caliceal stones associated with pain with similar success.

Why certain patients with immobile caliceal stones have pain remains enigmatic. The results of treatment are too consistent to result from placebo effect. The stones could cause infundibular spasm proximally and create distention of the calix, but this phenomenon was never witnessed on numerous IV and retrograde pyelograms which were performed primarily or as part of their percutaneous procedures. Many of the procedures were performed under local anesthesia, and in these cases the entry of the needle into the calix and even the presence of the retrograde catheter resulted in a great deal more pain than usually noted. Our assumption is that the caliceal stone acts as an irritant to the collecting system, thus affecting the way that stimuli through the intrinsic nerves of the kidney are carried through the sympathetic nervous system. This may result in abnormal muscular peristalsis in the renal collecting system and result in pain similar to that treated by renal denervation procedures. ${ }^{4,5}$ The description of the pain treated successfully by denervation is similar.

Complete removal of the stones by percutaneous methods had uniform success with the exception of 1 patient whose stone could not be accessed. Only a single patient treated with ESWL failed when a lower pole calix was treated and the fragments did not pass. Of the 11 patients treated with ESWL, 7 had lower caliceal stones. Only one stone failed to fragment satisfactorily and required subsequent percutaneous removal to achieve a stone-free and pain-free result. Thus, our recommendation is that ESWL is effective and may be preferred by most patients because of the lower morbidity. ${ }^{11}$ In addition, a percutaneous approach is rendered no more difficult should ESWL fail.

A basic principle of medicine is that the cure offered not be worse than the disease being treated. In the past, the short-term and longterm morbidity of flank exploration for caliceal stone removal was often greater than the severity of the patient's symptoms, thus making removal of these calculi inadvisable. While we do not advocate treatment of asymptomatic caliceal stones, the lowered morbidity of PSE and ESWL now makes the removal of nonobstructive caliceal stones associated with noncolicky pain appropriate in many circumstances. Although ESWL is significantly less costly per patient treatment than surgery, ${ }^{12}$ concern has been expressed about its overutilization for stones previously thought to be "nonsurgical."13 Even though expanding the indications for ESWL to the group of patients described in this article might increase health care expenditures for urolithiasis, this appears to be justified by the marked reduction in pain and rapid return to normal activity achieved.

\section{North Senate Blvd., Suite 655 Indianapolis, Indiana 46202}

(DR. LINGEMAN)

\section{References}

1. Chaussy, et al: Extracorporeal Shock Wave Lithotripsy. New Aspects in the Treatment of Stone Disease, Basel, S. Karger, 1982.

2. Dewolf WC, and Fraley E: Renal pain, Urology 6: 403 (1975).

3. Mitchell GA: The renal nerves, Br J Urol 22: 269 (1950).

4. Harris $\mathrm{H}$, and Harris $\mathrm{R}$ : Renal sympathetic stones, renal pain, and renal sympathectomy, Br J Urol 2: 367 (1930).

5. Laughton J: The function of the sympathetic nerve supply to one kidney and the effect of denervation, Br J Clin Prac 20: 353 (1972).

6. Nesebar R, Pollar J, and Fraley E: Renal vascular impressions, Am J Roentgen Med 101: 719 (1967).

7. Fraley E: Vascular obstruction of superior infundibular causing nephrologia, N Engl J Med 275: 1403 (1966).

8. Ray B, and Neill C: Abdominal visceral sensation in man, Ann Surg 126: 709 (1947).

9. Andersson $\mathrm{L}$, and Sylven $\mathrm{M}$ : Small renal calyceal calculi as a cause of pain, J Urol 130: 752 (1983).

10. Brannen GE, Bush WH, and Lewis GP: Calyceal calculi, J Urol 135: 1142 (1986).

11. Lingeman JE, et al: Comparison of results and morbidity of percutaneous nephrostolithotomy and extracorporeal shock wave lithotripsy, J Urol 138: 485 (1987).

12. Lingeman JE, Saywell RM, Woods JR, and Newman DN: Cost analysis of extracorporeal shock wave lithotripsy relative to other surgical and non-surgical treatment alternatives for urolithiasis, Med Care 24: 1151 (1986).

13. Mulley AG: Shock wave lithotripsy. Assessing a slam bang technology, N Engl. J Med 314: 845 (1987). 